\title{
FIRST EXPERIENCES WITH THE TRIMBLE SX10 SCANNING TOTAL STATION FOR BUILDING FACADE SURVEY
}

\author{
E. Lachat*, T. Landes, P. Grussenmeyer
}

ICube Laboratory, Photogrammetry and Geomatics Group, INSA Strasbourg, France (elise.lachat, tania.landes, pierre.grussenmeyer)@insa-strasbourg.fr

Commission II

KEY WORDS: Terrestrial Laser Scanning, Trimble SX10 scanning total station, UAV photogrammetry, building facade survey, georeferencing, data comparison

\begin{abstract}
:
The use of Terrestrial Laser Scanner (TLS) tends to become a solution in many research areas related to large scale surveying. Meanwhile, the technological advances combined with the investigation of user needs have brought to the design of innovative devices known as scanning total stations. Such instruments merge in a unique hardware both scanning and surveying facilities. Even if their scanning rate is often reduced compared to conventional TLS, they make it possible to directly georeference laser scanning projects and to complete them with measurements of individual points of interest. The recent Trimble SX10 which was launched on the market in early October 2016 has been tested and some experiences carried out with it are reported in this paper. The analyses mainly focus on the survey of a building facade. Next to laser scanning survey, a photogrammetry campaign using an Unmanned Aerial Vehicle (UAV) has been carried out. These different datasets are used to assess the Trimble SX10 issued point clouds through a set of comparisons. Since georeferencing is possible either directly or indirectly using this device, data processed both ways are also compared to conclude about the more reliable method.
\end{abstract}

\section{INTRODUCTION}

A review of the literature of the last decades shows that Terrestrial Laser Scanners (TLS) have been extensively used in geosciences, infrastructure monitoring and change detection of natural or artificial objects, among other research topics. As expected in the works of Böhler (2006) or Grussenmeyer et al. (2008), the field of heritage documentation follows the same trend and uses widely this technique today, as depicted by Stylianidis and Remondino (2016). As a matter of fact, TLS enable the accurate and fast acquisition of millions of points representing the surveyed scene. Driven by always more accurate and efficient sensor technology, as well as always deeper data processing software capabilities, laser scanning is nowadays accepted as a reliable method for accurate point cloud acquisition. Besides, investigation of TLS performances was a prominent topic of research in the last decades, so that a wide range of contributions about calibration (Lichti and Gordon, 2004), study of external parameter influences (Soudarissanane et al., 2011) but also performance analysis of devices (Hanke et al., 2006) are available.

In the field of conventional surveying, terrestrial laser scanning also sometimes becomes an alternative to total stations. Indeed, a large amount of points can be captured and the accuracy of produced data may be sufficient for some projects. However, depending on the kind of deliverables to produce, total station remains a highly used device. Besides, a total station is also required in laser scanning projects when the point clouds need to be known in a specific geodetic system, such as for example a national coordinate system. This process is known as indirect georeferencing. For those reasons, parallel to the emerging use of TLS in surveying projects, total station technology has known drastic changes. In about fifty years, the electronics have brought numerous new facilities to this kind of instrumentation, in such a way that total stations now tend to become multi-task devices composed of several built-in sensors (Scherer and Lerma, 2009).
In this context, modern total stations offering scanning facilities are nowadays marketed. Most of them only offer a low scanning rate compared to conventional TLS, given that pre-defined scanning modules like line or grid scanning are provided. These simple scanning functions reach a scanning rate lower than 20 points per second, which is also mainly due to the mass of the telescope reducing the rotating velocity of the hardware. Evgenikou and Georgopoulos (2015) relate on experiences of small artifact reconstruction with a Topcon IS Imaging Station, and the presented results illustrate the low point density which is reached while using such a combined device. The recent Trimble SX10 scanning total station launched on the market in early October 2016 offers a significant improvement in terms of scanning rate. With the capability of acquiring about 26,600 points a second, it can be described as a survey instrument merging high-speed 3D scanning and total station facilities.

Provided that some reference points are known, laser scanning projects performed using Trimble SX10 can be directly georeferenced. This may be of interest for the survey of building facades, once the produced models are intended for integration in a 3D city model for example. Georeferencing approaches are described in Reshetyuk (2009) and their respective benefits and drawbacks are mentioned. Regarding the final accuracy of georeferenced data, Lichti and Gordon (2004) report on the error budget related to direct georeferencing. Furthermore, it is also assumed in Reshetyuk (2009) that direct georeferencing may be rather adapted for outdoor surveys with large spatial extent. Given that both georeferencing methods are achievable with the Trimble SX10, a first aim of this paper is to compare both approaches using the case study of a church facade survey. The same dataset was also captured using various devices and techniques, which are firstly presented. Comparisons between these different datasets are then established in a dedicated section in order to assess the precision of the Trimble SX10 issued point clouds.

\footnotetext{
${ }^{*}$ Corresponding author
} 


\section{MATERIALS}

\subsection{Terrestrial Laser Scanners}

Aim of this paper is to assess the geometrical accuracy of point clouds acquired using Trimble SX10 scanning module. For this purpose, similar acquisitions have been carried out with a second TLS in order to produce a reference dataset.

2.1.1 Trimble SX10 scanning total station is a recent device intended for use in laser scanning projects as well as for survey of individual points of interest. All the conventional coordinate geometry (COGO) computations available in total stations are present within this instrument. This means that Trimble SX10 can be set up and centered on a known point, and oriented on references. While preparing a laser scanning project, the captured point clouds can thus be directly known in the reference system, provided that some survey points are available in the project area. This operation is known as direct georeferencing. Trimble SX10 has been designed to allow the user to carry out only one unique device in the field, which is one of its major advantages. As a matter of fact, a laser scanning project can easily be completed thanks to the measurements of individual points of interest using the total station facilities.

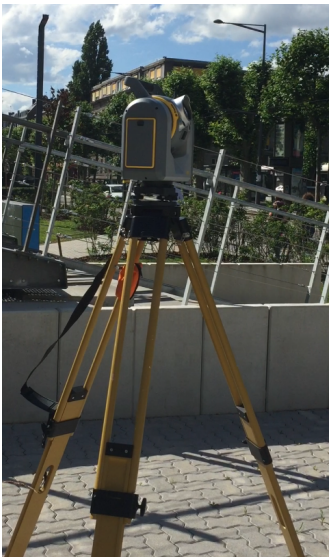

(a)

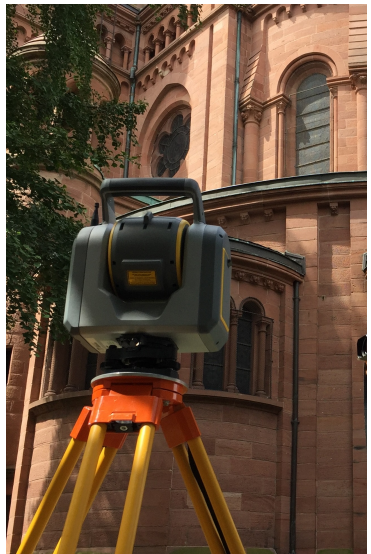

(b)
Figure 1. (a) Trimble SX10 scanning total station placed on a tripod and centered on a reference point. (b) View of the rear side of the device.

Next to the combination of geodetic survey and scanning facilities, several cameras used for various purposes are mounted on Trimble SX10 device. The absence of telescope on the hardware mainly contributes to its originality. As a matter of fact, optical sighting is no more foreseen and this operation is replaced by the use of the on-board camera system. Thus the hardware is remotely steered thanks to a tablet connected to the device, since no screen is present on the hardware. This specificity is visible in Figure $1 b$ showing the rear side of the device. Three cameras with different fields of view are available, providing three different zoom levels for aiming at a point. The overview camera is first applied for progressive zoom in, then the primary camera and finally the telescope camera, which offers the greater magnification. For point cloud colorization, the user can choose between the two first cameras and the overlap between pictures can be selected. These cameras are also useful for the creation of panoramas or for the documentation of the project, such as the documentation of reference point location for example. Compared to a conventional camera, this imaging system makes it possible to geolocalize the acquired panoramas and images, provided that the instrument has been set up on a known point. Moreover, centering the device is also achieved using a fourth camera known as plummet camera.

2.1.2 Trimble TX8 is the terrestrial laser scanner which has been used in order to acquire a reference dataset for the following data comparisons. Based on the same time-of-flight measurement technology as Trimble SX10 scanning module, some main technical specifications of both laser scanning systems are presented in Table 1, whereas SX10 is also a total station and imaging system.

\begin{tabular}{|l|c|c|}
\cline { 2 - 3 } \multicolumn{1}{c|}{} & Trimble TX8 & Trimble SX10 \\
\hline Range & $120 \mathrm{~m}$ & $600 \mathrm{~m}$ \\
Scanning rate & 1 million pts $/ \mathrm{sec}$ & $26,600 \mathrm{pts} / \mathrm{sec}$ \\
Field of view & $360^{\circ} \times 317^{\circ}$ & $360^{\circ} \times 300^{\circ}$ \\
& Range syst. & Distance meas. \\
Accuracy criterion* & error: $<2 \mathrm{~mm}$ & accuracy: $2 \mathrm{~mm}$ \\
& from $1.9 \mathrm{~mm}$ to & from $1 \mathrm{~mm}$ to \\
Point spacing & $15.1 \mathrm{~mm} @ 10 \mathrm{~m}$ & $10 \mathrm{~mm} @ 10 \mathrm{~m}$ \\
\hline
\end{tabular}

* terminology according to manufacturer datasheets

Table 1. Overview of some technical properties of the two laser scanners used in this study, according to manufacturer specifications

\subsection{UAV photogrammetry}

The Unmanned Aerial Vehicle (UAV) employed to perform picture acquisitions on this facade is the Albris from Sensefly company, which has been initially designed for close range inspection. The imaging sensor has a resolution of $7152 \times 5368$ pixels for a sensor size of $10 \times 7.5 \mathrm{~mm}$, and the lens has a focal length of $8 \mathrm{~mm}$. Due to these specifications, a Ground Sampling Distance (GSD) of $0.9 \mathrm{~mm}$ at 5 meters is achievable.

\section{DATA ACQUISITION AND PROCESSING}

This section gives an overview of the different acquisition protocols which have been applied and presents the datasets produced.

\subsection{Facade under study}

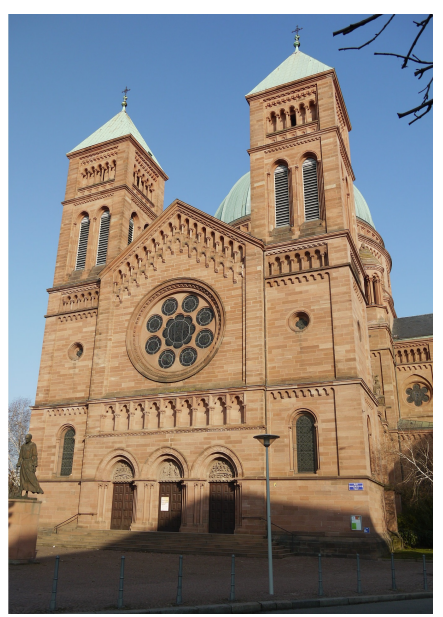

Figure 2. Main facade of the Saint-Pierre-le-Jeune Catholic Church (Strasbourg, France) 
For this study, datasets have been acquired on the facade of a church located near the city center of Strasbourg, France. The Catholic Church Saint-Pierre-le-Jeune was built at the end of the 20th century in the neo-Romanesque style, and it is made of red and pink sandstone as visible in Figure 2. Due to its architectural style, the facade contains detailed parts but also planar surfaces, which are of interest for the presented investigation.

\subsection{Laser scanning survey}

In order to avoid the presence of too much masks and thus holes on the facade, the laser scanning survey has been performed from three different points of view as shown in Figure 3. In spite of the possibility to perform direct georeferencing of point clouds, Trimble SX10 scanning module can also be used alone as a common TLS to perform free scanning stations. However a network of survey points available in the church surroundings has been used during measurement campaign with Trimble SX10 device. By this means, the coordinates of the three scanning stations have been determined, leading to the acquisition of three directly georeferenced point clouds of the whole facade. In this case, theoretically no post-processing steps such as point cloud registration are required given that individual point clouds are already oriented, with final point coordinates. Some benefits and drawbacks of direct and indirect georeferencing of point clouds are discussed in the next section, based on the example of the results obtained for this case study.

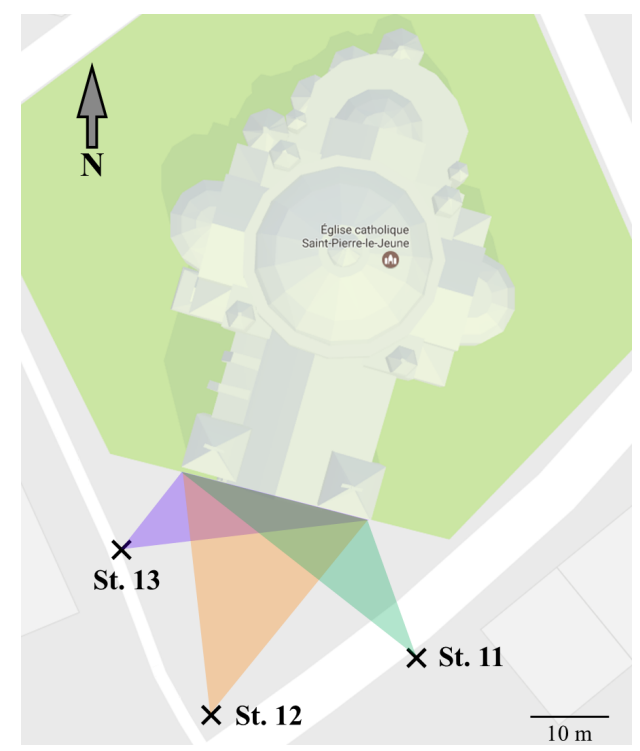

Figure 3. Location of the three scanning stations in front of the facade (map from Google Maps, modified)

Regarding measurement settings, four different point spacing values can be chosen on SX10 device from $1 \mathrm{~mm}$ at $10 \mathrm{~m}$ to $10 \mathrm{~mm}$ at $10 \mathrm{~m}$. A point spacing of $2 \mathrm{~mm}$ at $10 \mathrm{~m}$ was used during acquisitions from stations 11 and 12 (see Figure 3) located at about 15 to $25 \mathrm{~m}$ of the facade. For the third station which is closer to the facade, a point spacing of $5 \mathrm{~mm}$ at $10 \mathrm{~m}$ was selected. A mean point spacing of about $5 \mathrm{~mm}$ is achieved for the whole SX10 point cloud, which counts about 13 millions of points after the segmentation of the facade under study. A further advantage of Trimble SX10 scanning interface is the possibility to directly define a frame where point cloud has to be captured, without the necessity to perform a $360^{\circ}$ preview acquisition beforehand.
Acquisitions with the Trimble TX8 laser scanner have been carried out from free scanning stations which were chosen near the previously mentioned stations. Since TX8 laser scanner can not be centered on a known point, locations of the stations slightly differ. However these stations ensure a low variation of the scanning angles between both devices. Due to the point spacing of $5.7 \mathrm{~mm}$ at $30 \mathrm{~m}$ chosen during this second measurement campaign, this second dataset is more dense than the SX10 point cloud. The registration of TX8 point clouds has been achieved using the plan-based registration algorithm available in the manufacturer software Trimble RealWorks. Remaining deviations between individual scans after registration are very low (less than 2 $\mathrm{mm})$.

\subsection{Processing of images from UAV}

While dealing with aerial photogrammetry, the flight plan has to be carefully prepared. To perform acquisitions on this church facade, a flight distance of about $8 \mathrm{~m}$ has been chosen. A theoretical pixel size (or GSD) of about $1.4 \mathrm{~mm}$ on the surveyed object is expected while flying at this distance. Moreover to complete the standard perpendicular images, further oblique images have been acquired on the facade, leading to a better observation of detailed areas. More information about acquisition protocol applied during this survey campaign can be found in Murtiyoso et al. (2017).

Regarding image processing in order to obtain dense point clouds, many dense matching algorithms were developed in the past years, and this is still a prevailing topic of research. These algorithms are implemented within several automatic or semi-automatic software solutions useful for picture orientation, and point cloud triangulation and densification. Thus a wide range of commercial or open source solutions are available to solve these computations. The commercial software Photoscan from Agisoft has been applied to compute the dense point cloud used within this study. A medium quality criterion was chosen to generate the dense point cloud, because of the computation time related to the number of pictures. Using the same set of images, experiments for point cloud computation through dense matching have been performed by means of three more software solutions. The results of these processes are reported by Murtiyoso et al. (2017).

\section{COMPARISON BETWEEN DIRECT AND INDIRECT GEOREFERENCING}

Given that both georeferencing methods can be achieved while working on a laser scanning project with Trimble SX10, they are compared based on this particular case study. Using the network of known points located in the church surroundings, the three individual point clouds captured with the SX10 device and covering the church facade have been directly georeferenced during the measurement campaign. The accuracy of this direct georeferencing is evaluated throughout the two following subsections.

Indirect georeferencing of these point clouds was also carried out in order to produce a second dataset, even if the remaining transformations were quite low. To this end, the alignment of the individual point clouds was refined by means of an ICPlike algorithm which delivered a final alignment error of about $1 \mathrm{~mm}$. Georeferencing was then performed by picking some known points in the registered point clouds. Figure 4 shows some Ground Control Points (GCP) which are evenly distributed on the facade and were previously measured using a Leica TS02 total station. Based on $11 \mathrm{GCP}$ which were manually selected in the registered data, a georeferencing error of about $1 \mathrm{~cm}$ was achieved. These two post processing steps were carried out using the manufacturer software Trimble RealWorks. 


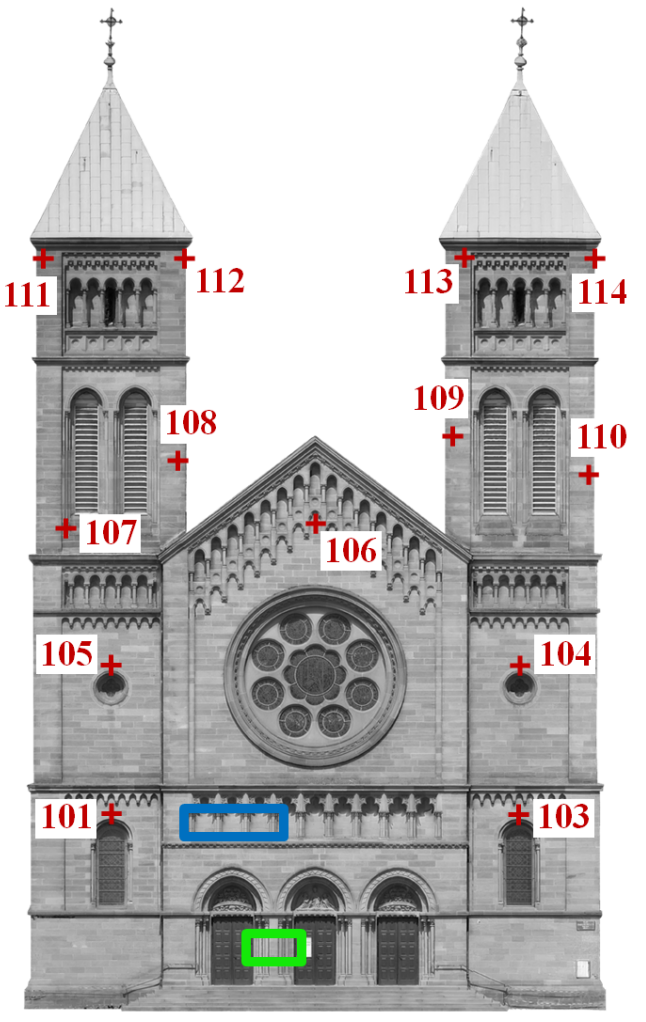

Figure 4. Distribution of the Ground Control Points (GCP) on the facade. Blue and green rectangles highlight architectural elements used for comparisons in next sections.

\subsection{Georeferencing assessment based on the analysis of GCP}

Once directly as well as indirectly georeferenced datasets are available, their comparison is achieved trough the analysis of the 3D coordinates of GCP in each dataset. The GCP visible in the point clouds have been manually picked, and the measured coordinates have been compared to the known coordinates of these points used as ground truth. Thus the 3D deviations listed in Table 2 are computed. Points have been measured in each individual point cloud in the case of direct georeferencing, whereas the registered point clouds in the second case are considered as a unique entity during point selection.

The mean spatial deviations documented in this table show that indirect georeferencing is more accurate than direct georeferencing in the considered case study. A mean 3D deviation of 1.4 $\mathrm{cm}$ is computed based on GCP for the indirectly georeferenced point cloud, which is lower than for each of the individual point cloud resulting from the first method. Depending on the scanner location, some points are not present in individual point clouds as visible with the empty lines in table. However while using only the minimum set of points which are common to each column, the mean deviations remain in the same order of magnitude as presented here. It should be noticed that picking errors made by operator while manually selecting the GCP on the different point clouds influence these deviations, however they are in the same order of magnitude for each considered point cloud since operator as well as data characteristics did not change during the project.

Even if direct georeferencing is a quite convenient method since it does not require any post processing treatments after acquisition campaign, it may not always be the better solution in regards of final model accuracy. This empirical analysis based on the particular case study presented here has been confirmed many times in

\begin{tabular}{|l|c|c|c|c|}
\hline \multirow{2}{*}{ Points } & \multicolumn{2}{|c|}{ Direct georeferencing } & \multicolumn{1}{c|}{$\begin{array}{c}\text { Indirect } \\
\text { georeferencing }\end{array}$} \\
\cline { 2 - 4 } & St. 11 & St. 12 & St. 13 & 1 \\
\hline 101 & 2.3 & 3 & 0.7 & 0.2 \\
103 & 1.1 & 2.8 & 1.3 & 1.2 \\
104 & 1 & 3.3 & 2.3 & 0.5 \\
105 & 2.5 & 2 & 1.5 & 0.6 \\
106 & 2.2 & 3.9 & 0.9 & 1 \\
107 & 3.7 & 4.3 & 1.8 & 1.7 \\
108 & 4.3 & 4 & 3.7 & 0.8 \\
109 & 3.7 & 5 & n.a. & 1.8 \\
110 & 2.5 & 2.6 & n.a. & 2.1 \\
111 & 2.9 & 4.2 & 4.5 & 3.2 \\
112 & 3.6 & 5.9 & 2.9 & 2.4 \\
113 & 2.9 & 5 & n.a. & 1.3 \\
114 & n.a. & 4.6 & n.a. & $\mathbf{1 . 4} \mathbf{~ c m}$ \\
\hline Mean & $\mathbf{2 . 7} \mathbf{~ c m}$ & $\mathbf{3 . 9} \mathbf{~ c m}$ & $\mathbf{2 . 2} \mathbf{~ c m}$ & \\
\hline
\end{tabular}

Table 2. 3D deviations (absolute values, in $\mathrm{cm}$ ) computed on GCP for directly georeferenced point clouds, and indirectly georeferenced whole point cloud

the literature, such as by Alba and Scaioni (2007) and by Schuhmacher and Böhm (2005). In the following subsection, directly georeferenced data of the considered facade are more finely analyzed in order to highlight the potential effects of such a method on data quality.

\subsection{Direct georeferencing closer overview}

As previously mentioned, the three SX10 laser scanning point clouds of the facade have been directly georeferenced in the field. Due to a bad visibility in this urban scene, the computation of scanning station coordinates through resection was not possible for each of them since sometimes less than three references were visible. Station coordinates were thus determined beforehand and station orientation was performed on the visible references before scanning. Obviously this configuration is not the more favorable for the survey. For these reasons a particular attention is paid to georeferencing precision afterward. A closer look at the raw dataset shows that the alignment of the individual point clouds is not perfect. To illustrate this unwanted effect, a horizontal crosssection going through the blind arcade pillars located above the tympana (see blue rectangle in Figure 4) is studied. The thin slice performed in the directly georeferenced point clouds is shown in Figure 5a. A deviation which can achieve about $3 \mathrm{~cm}$ is clearly visible between these point clouds. On the contrary the indirectly georeferenced point clouds perfectly overlap as illustrated in Figure $5 \mathrm{~b}$, due to the alignment refinement carried out.

In the context of this survey, the cross-sections in Figure 5 underline one of the potential weaknesses of performing direct georeferencing, namely the bad overlap between individual data which may occur. It should be noticed that the performed geodetic measurements may be involved in the results obtained for direct georeferencing. This has probably increased the displacements between individual point clouds. Nevertheless, even if performing direct georeferencing is quite intuitive once the hardware makes it possible, a careful attention should be paid to the survey methodology. If the network configuration does not seem to be favorable, the need for data post-processing related to indirect georeferencing should not bypass the application of this latter approach. A further argument for indirect georeferencing of registered point clouds is the high number of efficient registration 


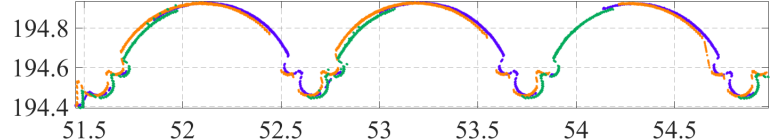

(a)

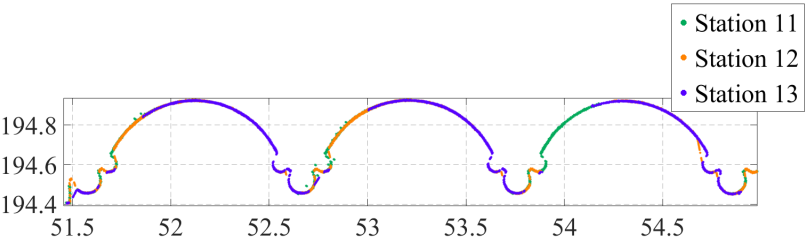

(b)

Figure 5. (a) Horizontal cross-section performed in SX10 directly georeferenced point clouds, above the three tympana. (b) Same section carried out in the aligned point clouds after indirect georeferencing. Scales are in meters.

algorithms which are available. As a matter of fact, numerous automated algorithms are implemented in the different point cloud processing software. They can rely on target or spheres detection and matching, but more and more they are also dealing with feature-based matching such as plan-based registration, which are methods borrowed from the computer sciences. Thus depending on the project, the registration of individual point clouds before the complete project georeferencing may be a better way to process, avoiding the presence of errors due to direct georeferencing of each scan.

In conclusion, a careful preparation of the survey and the analysis of the requirements in terms of data to be produced are of high importance before measurement campaign. Once a laser scanning project needs to be georeferenced, Trimble SX10 scanning total station presents the advantage of requiring only one device in the field, whether georeferencing is performed directly or indirectly. Even if direct georeferencing of scanning projects is obviously less accurate than the indirect approach, it can provide useful initial location and alignment of the point clouds which can then be easily refined through automated algorithms such as ICP.

\section{RESULTS OF DATA GEOMETRIC EVALUATION}

In this section, the geometrical accuracy of point clouds produced using Trimble SX10 scanning module is investigated. To mainly concentrate on the data quality and to avoid the influence of external parameters, georeferencing is no longer taken into account.

\subsection{Evaluation of the Trimble SX10 laser scanning survey}

A first step consists in the quality assessment of the produced point clouds through a set of comparisons performed with a reference dataset. Acquisitions carried out with the Trimble TX8 laser scanner as explained in Subsection 3.2 are used to this end. The three registered reference point clouds have been meshed using the Poisson surface reconstruction algorithm available in the free software CloudCompare. This will be of interest to compute signed deviations between both datasets.

5.1.1 An overall comparison is firstly performed, taking into account the whole facade with the two towers. Both datasets have been finely aligned beforehand using an ICP-like algorithm, leading to a final alignment error slightly smaller than $2 \mathrm{~cm}$. The dedicated CloudCompare software is then used to compute the cloudto-mesh deviations. Result of these computations is shown in
Figure 6, whereby deviations are projected on the Trimble SX10 point cloud. For a better visualization of the signed deviations, these have been filtered in a range from $-1.5 \mathrm{~cm}$ to $1.5 \mathrm{~cm}$ since more than $99.9 \%$ of the points are present in this interval. One can thus assume that no significant information is lost through this filtering. Besides, elements which were different between both acquisition campaigns have been segmented in order to remove their incidence on the statistical value computation. These elements are the main gate which was opened in one case and closed in the other, and a rectangular banner placed above the three tympana while the Trimble SX10 scanning survey was performed.

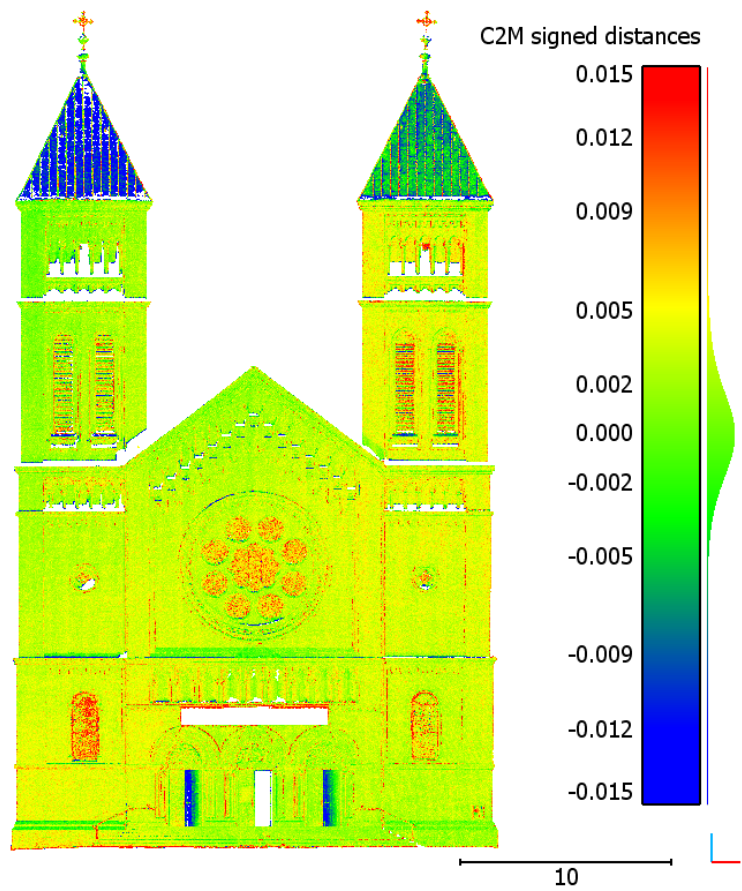

Figure 6. Cloud-to-mesh deviations computed between the meshed point cloud issued by Trimble TX8 and the Trimble SX10 whole point cloud. Deviations (in meters) are projected on SX10 point cloud.

Both datasets present very similar geometries since a mean deviation of $0.1 \mathrm{~mm}$ is observed, with a standard deviation of about 3 $\mathrm{mm}$. The highest deviations are visible on the roofs made of copper as well as on the two non segmented gates. This is probably due to the materials used for these elements, which have an influence on the way laser returns are handled within both scanning devices. Deviations also appear on the stained-glass windows where measurement noise is generally present because of glass properties. Nevertheless the deviations caused by the noise on these elements do not exceed $1 \mathrm{~cm}$. Except on surfaces with particular properties, no significant deviations with respect to data coming from another TLS are noticed. Hence the reliability of Trimble SX10 in this scanning project can be validated. To confirm the statistical values derived from this overall analysis, individual detailed areas of the facade are investigated hereafter.

5.1.2 A comparison of detailed parts is then carried out in order to assess the level of detail achieved thanks to SX10 laser scanning module. Areas selected for the finer investigations are the central tympanum and the rose window. Reflective characteristics of windows may be unadapted for processing and assessing laser scanning data, nevertheless this second architectural el- 
ement is not only made of glass and contains stone-made carved contour and details which are of interest. Close-up views of the cloud-to-mesh distances computed on these particular areas are presented in Figure 7 for a better visualization of the deviations affecting them.

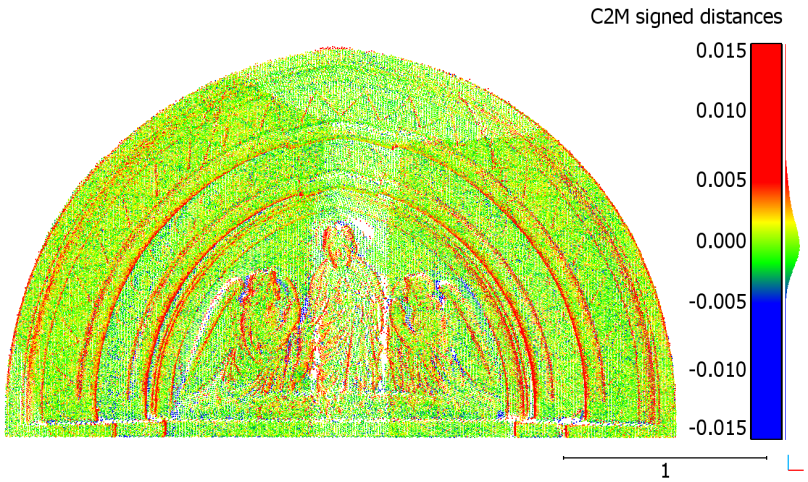

(a)

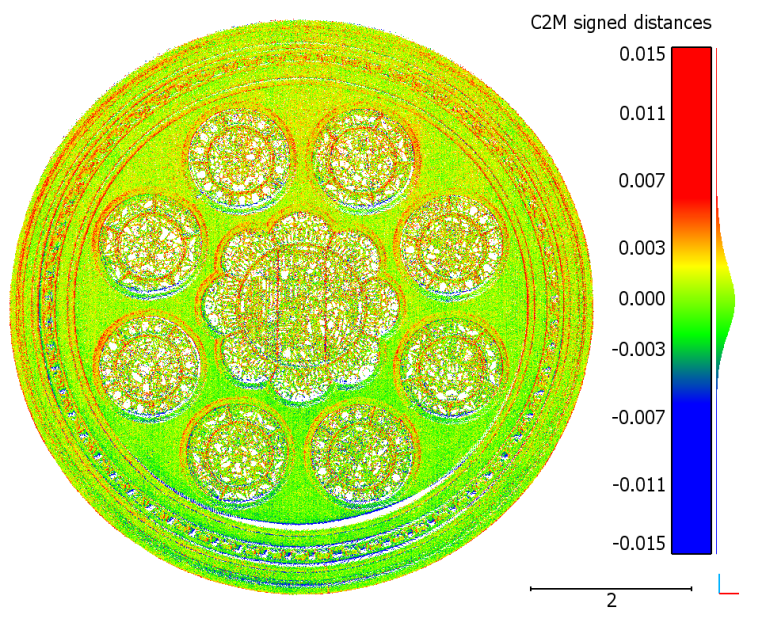

(b)

Figure 7. Cloud-to-mesh deviations (in m) observed on detailed parts and projected on Trimble SX10 point cloud: (a) central tympanum and (b) rose window

Based on these comparisons, similar statistic values as those derived from the overall comparison are observed. For both elements, the mean deviation is smaller than $1 \mathrm{~mm}$ (respectively 0.5 $\mathrm{mm}$ for tympanum and $0.2 \mathrm{~mm}$ for rose window). With a value which is slightly smaller than $3 \mathrm{~mm}$, the standard deviation is similar for both artefacts, but also coherent with the whole facade survey. Only boundaries of the statues or moldings are affected by the deviations, since on these locations the laser beam hits the surface at a grazing angle of incidence. The high level of detail achieved in the point clouds produced using Trimble SX10 scanning total station is also highlighted by these closer comparisons. As a matter of fact, the carved parts in stone but also the fine iron structure of the stained-glass windows of the rose are clearly visible in these point clouds. The comparisons carried out in this subsection allow us to conclude about the high precision of the produced dataset.

\subsection{Comparison with photogrammetry-based model}

To complete this study, an additional dataset of the considered facade was used. The available data consists in a point cloud computed through the dense matching of pictures acquired by a
UAV. The overall assessment of this point cloud is not the topic of this paper and such an analysis is performed by Murtiyoso et al. (2017). This is the reason why only characteristic areas of the facade are used in this last section, to compare both datasets based on cross-sections and noise analysis.

5.2.1 Detailed areas represented by two rectangles in Figure 4 have been selected. The first area is located between two gates (green rectangle), whereas the second one is located on blind arcades containing pillars above the three tympana (blue rectangle). These architectural elements present round surfaces but also right-angled corners in particular for the sculpted decor between the gates. Cross-sections have been carried out in the point cloud issued by Photoscan, as well as in both laser scanning point clouds. To avoid the visualization problems related to the drawing of vectorized profiles, the results are presented in form of thin point cloud slices (between 1 and $3 \mathrm{~cm}$ thickness, depending on point cloud density) in Figure 8.

A first observation is the high correlation between cross-sections derived from Trimble TX8 and SX10 devices. Their perfect overlap confirms the high level of precision for SX10 data as stated from the previous cloud-to-mesh comparisons. More generally, in these two sections the distribution of points seems to follow the shape of element profiles. Regarding the cross-sections from photogrammetry-based point cloud, another phenomenon is observed. It is clearly visible in Figure 8a that protruding edges are largely rounded. In both sections, even the curved surfaces are smoothed and thus original diameter of the pillars is not respected. However these sections performed in the point cloud computed with Photoscan show a high degree of completeness since they are more or less continuous along the shape. The absence of holes even on hardly reachable areas is probably due to the computation of extrapolated points carried out during dense matching process. This effect is not necessarily observed while processing dense matching computations with other software solutions such as PhotoModeler or MicMac, for which the resulting point clouds rather follow the true geometry of objects. In the presented example, one can assume that these results are mainly correlated with the software solution used, as was already illustrated by Murtiyoso et al. (2016).

5.2.2 The noise present in point clouds is then evaluated based on planar surfaces. The same planar areas on the left and right parts of the facade have been segmented in both Trimble SX10 and dense matching point clouds. The standard deviation values computed on planes adjusted in both datasets reach between $2.8 \mathrm{~mm}$ and $3.0 \mathrm{~mm}$ in both cases. It should be noticed that the noise observed in these surfaces is not only due to surveying techniques. This noise is also related to the facade material since the texture of stones is not perfectly smooth. Regarding the obtained values of standard deviation which are very close, one can assume that a filtering is applied during Photoscan processing operations.

In conclusion to these analyses, it appears in the considered study that the level of detail achieved is higher using laser scanning in spite of some holes in parts hardly reachable by the laser beam. Indeed the geometry of architectural elements is better respected in the laser scanning survey than in the dense matching point cloud computed using Photoscan. This latter point cloud is very complete and does not contain holes but this is due to internal treatments which are made at the expense of precision and reliability of the final data. This has been illustrated by sections in the resulting point cloud, where edges and details are largely smoothed. 


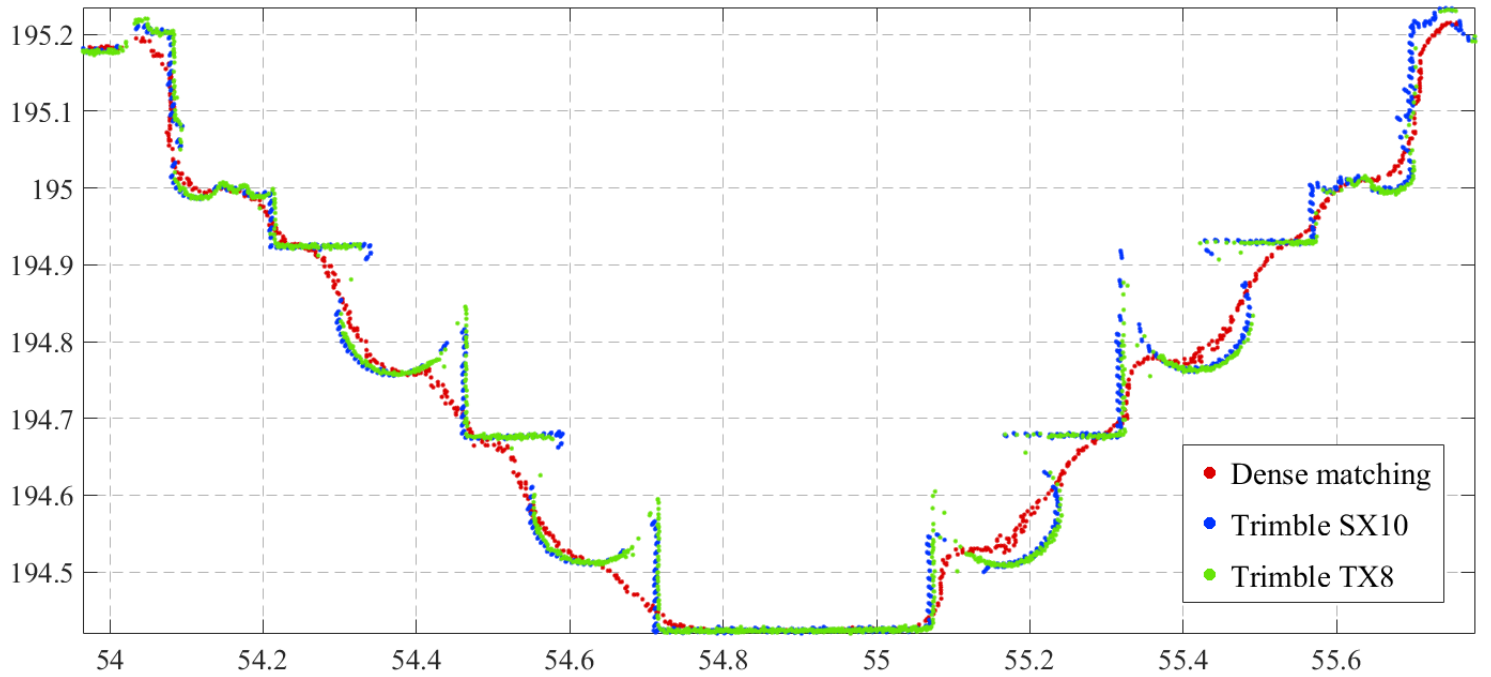

(a)

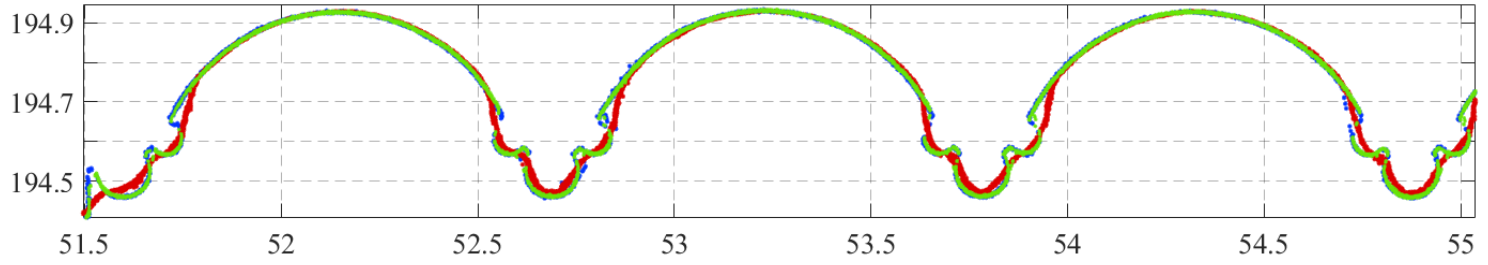

(b)

Figure 8. (a) Cross-sections located on the carved decor between gates and performed in Trimble SX10 point cloud (in blue) and in dense matching point cloud from Photoscan (in red). Reference section (in green) is issued from TX8 laser scanner point cloud. (b) Cross-sections performed in the same point clouds and located on the pillars above tympana. Scales are in meters.

\section{CONCLUSION}

The recently marketed Trimble SX10 scanning total station was introduced in this paper based on the case study of a church facade survey. The point clouds acquired thanks to its scanning module can be directly georeferenced during the field work, and they can be completed by further measurements of individual points of interest. The specific design of its hardware results in an innovative and versatile survey instrument.

The acquisitions carried out have been confronted in different ways, starting with the investigation of direct georeferencing in comparison with indirect approach. Some potential weaknesses while performing direct georeferencing have been underlined based on the example of a facade survey, and it appears that indirect georeferencing delivers more accurate results. It has also been noticed that the results obtained are highly correlated with the configuration of reference network, its intrinsic accuracy, as well as with the survey methodology applied. Nevertheless, regarding the use of Trimble SX10 in this context, the need to carry out an additional total station for georeferencing is avoided. Moreover, georeferencing with SX10 device is performed through conventional geodetic measurements. The tools available for direct georeferencing are largely improved compared to the standard method once using TLS, where targets need to be scanned with a specific density.

Then the geometrical precision of the produced point clouds was assessed without consideration of georeferencing. The scanning module of Trimble SX10 has proved to be as reliable as another TLS, even for capturing detailed parts. Further comparisons with a point cloud computed through dense matching have shown that the geometry of architectural elements is better respected in the laser scanning survey, even if these results are probably correlated with the dense matching software used to compute this second dataset. The aforementioned comparisons illustrate the reliability of the data produced with Trimble SX10 for laser scanning survey as well as for geodetic works. Regarding the advantages offered by the device, it will be interesting to consider the datasets produced with it in other case studies in the future.

\section{ACKNOWLEDGEMENTS}

The authors would like to thank the Trimble company for borrowing the SX10 scanning total station in the frame of a beta-test program. They also want to thank Arnadi Murtiyoso for providing the dense matching point clouds derived from UAV acquisitions and performed during its master project.

\section{REFERENCES}

Alba, M. and Scaioni, M., 2007. Comparison of techniques for terrestrial laser scanning data georeferencing applied to 3D modeling of cultural heritage. In: The International Archives of the Photogrammetry, Remote Sensing and Spatial Information Sciences, Vol. XXXVI-5/W47, Zurich, Switzerland, 8 pages.

Böhler, W., 2006. Comparison of 3D laser scanning and other 3D measurement techniques. In: E. Baltsavias, A. Grün, L. Van Gool and M. Pateraki (eds), Recording, Modeling and Visualization of Cultural Heritage, Taylor \& Francis Group, London, pp. 89-99. 
Evgenikou, V. and Georgopoulos, A., 2015. Investigating 3D reconstruction methods for small artifacts. In: The International Archives of the Photogrammetry, Remote Sensing and Spatial Information Sciences, Vol. XL-5/W4, Avila, Spain, pp. 101-108.

Grussenmeyer, P., Landes, T., Voegtle, T. and Ringle, K., 2008. Comparison methods of terrestrial laser scanning, photogrammetry and tacheometry data for recording of cultural heritage buildings. In: The International Archives of the Photogrammetry, Remote Sensing and Spatial Information Sciences, Vol. XXXVIIB5, Beijing, China, pp. 213-218.

Hanke, K., Grussenmeyer, P., Grimm-Pitzinger, A. and Weinold, T., 2006. First experiences with the Trimble GX scanner. In: The International Archives of the Photogrammetry, Remote Sensing and Spatial Information Sciences, Vol. XXXVI-5, Dresden, Germany, 6 pages.

Lichti, D. and Gordon, S. J., 2004. Error propagation in directly georeferenced terrestrial laser scanner point clouds for cultural heritage recording. In: Proceedings of the FIG Working Week, Athens, Greece, pp. 1-16.

Murtiyoso, A., Grussenmeyer, P. and Freville, T., 2017. Close Range UAV Accurate Recording and Modeling of St-Pierre-leJeune Neo-Romanesque Church in Strasbourg (France). In: The International Archives of the Photogrammetry, Remote Sensing and Spatial Information Sciences, 3D-ARCH Workshop 2017, Nafplio, Greece, 8 pages.

Murtiyoso, A., Grussenmeyer, P., Koehl, M. and Freville, T., 2016. Acquisition and Processing Experiences of Close Range UAV Images for the 3D Modeling of Heritage Buildings. In: Digital Heritage - Progress in Cultural Heritage: Documentation, Preservation, and Protection - Proceedings of 6th International Conference EuroMed 2016, pp. 420-431.

Reshetyuk, Y., 2009. Self-calibration and direct georeferencing in terrestrial laser scanning. PhD thesis, Royal Institute of Technology (KTH), Stockholm, Sweden.

Scherer, M. and Lerma, J. L., 2009. From the Conventional Total Station to the Prospective Image Assisted Photogrammetric Scanning Total Station: Comprehensive Review. Journal of Surveying Engineering 135(4), pp. 173-178.

Schuhmacher, S. and Böhm, J., 2005. Georeferencing of Terrestrial Laser scanner Data for Applications in Architectural Modeling. In: The International Archives of the Photogrammetry, Remote Sensing and Spatial Information Sciences, Vol. XXXVI5/W17, Venice, Italy, 7 pages.

Soudarissanane, S., Lindenbergh, R., Menenti, M. and Teunissen, P., 2011. Scanning geometry: Influencing factor on the quality of terrestrial laser scanning points. ISPRS Journal of Photogrammetry and Remote Sensing 66(4), pp. 389-399.

Stylianidis, E. and Remondino, F. (eds), 2016. 3D Recording, Documentation and Management of Cultural Heritage. Whittles Publishing, 388 pages. 